poorly to chemotherapy. Treatment of a relapse is similar to that of the original attack, but tests of cure should be even more meticulous.

\section{Summary of Treatment}

1. Make sure of the diagnosis.

2. Before starting chemotherapy inquire for history of previous intolerance. No special diet is necessary.

3. Give sulphathiazole, $5 \mathrm{~g}$. a day for 2 to 4 days, in evenly spaced doses; the drug need not be taken during the night. Give concurrent alkaline mixture and plenty of fluids. In place of sulphathiazole the following may be used: sulphadiazine, $6 \mathrm{~g}$. a day for 4 days ; sulphapyridine, $5 \mathrm{~g}$. a day for 5 days; or sulphanilamide, $5 \mathrm{~g}$. a day for 5 days. Never give a sulphonamide preparation for more than 7 consecutive days in any circumstances.

4. Look out for toxic effects of sulphonamides.

5. Look out for complications.

6. Always do a blood count before a second course of a sulphonamide.

7. Make certain that tests of cure are meticulously carried out : you cannot be sure the patient is cured unless you do. If he is not, he may unwittingly infect some innocent person.

\section{Warnings}

Cases are divided into two classes-those which are cured by sulphonamides and those which are not. Refer the latter to a specialist. You may regret it if you don't.

Don't give more or less of the sulphonamide than the dosage indicated above. If you overtreat, serious reactions which may endanger the life of the patient may occur. If you undertreat you may make the patient sulphonamide-resistant: he may then take months to cure.

Don't attempt procedures with which you are not familiar -e.g., irrigation, instrumentation, prostatic massage.

Don't use a syringe for intra-urethral treatment, and don't use provocative instillations for test of cure.

Gonorrhoea is a serious disease, and sulphonamides are dangerous drugs, not to be prescribed lightly.

The average V.D. patient is an unsatisfactory one; he is usually a liar. Tread very warily, especially as regards prognosis.

\section{Appendix : Technique of Irrigation}

Tell the patient to pass water.

Cleanse the glans penis with a little of the lotion to be used.

Use an irrigating vessel, preferably of glass, connected by six feet of rubber tubing to a blunt-pointed glass nozzle (Janet type).

Pour two pints of the lotion at $100^{\circ} \mathrm{F}$. into the container and expel air from tube and nozzle. Clip the tubing.

Adjust the height of the irrigating vessel not more than $2 \frac{1}{2}$ feet above the patient's penis.

Patient should stand, or lie on an examination table.

Insert nozzle into the meatus, release the clip, and allow lotion to fill the anterior urethra. Repeat three or four times, allowing the urethra to empty after each filling.

Insert nozzle and tell patient to relax, take deep breaths, and try to pass water. In this way lotion may be coaxed into the bladder. Don't force it.

Repeat this procedure two or three times.

\section{GONORRHOEA IN THE FEMALE}

Treatment of this is carried out on similar lines to that in the male. Chemotherapy in proportionate dosage, making allowance for weight, is usually successful provided there are no closed foci-e.g., bartholinitis. Douching is to be deprecated; dry swabbing or swabbing with weak alkali is usually preferable. Skenitis, bartholinitis, cervicitis, and salpingitis should be dealt with as they occur, preferably by a specialist. Tests of cure should be carried out immediately after the menstrual period for 3 consecutive months following termination of treatment. They should include both microscopical and bacteriological examination of all pathological specimens and, of course, "blood tests.

\section{Medical Memoranda}

\section{Intestinal Obstruction after Caesarean Sectio.. for Contracted Pelvis}

Cases of intestinal obstruction after Caesarean section reported up to 1938 are not numerous. O'Connor (1934) had one death from intestinal obstruction in 436 cases of Caesarean section, the patient dying on the sixth day. No suggestion was made as to the aetiology, except perhaps toxaemia. Haultain et al. (1933-4) found that irregular action of the bowels was present in 24 patients $(14.1 \%)$. The lax post-partum abdominal musculature, arising de novo, was the only aetiological agent suggested. Montgomery (1936) considered peritoneal adhesions a possible cause of intestinal obstruction after Caesarean delivery, but he does not mention the possibility of pelvic impaction of the uterus. Quigley (1937) reported three cases of intestinal obstruction in a total of 358 Caesarean sections simply as "ileus," with one case- - Intestinal Obstruction: Operation."

The following two cases, treated shortly after each other in 1930-1, aroused my interest at the time. Search of the literature, however, failed to disclose reports of any similar case, although some must surely have occurred. Both women were primiparae under 30. Gestation had been normal so far as was known; there was no evidence of toxaemia and no history of renal disease. In neither had there been any previous laparotomy, and no abnormality in the serous layers was observed at operation. Both were unbooked cases, admitted with obstructec labour.

CASE I

A. B., aged 19. Admitted to hospital on July 26, 1930, five hours in labour. No descent; Müller's test showed marked overlap of the anterior symphysial border; P.V., sacral promontory readily felt. Classical Caesarean section was performed the same day under

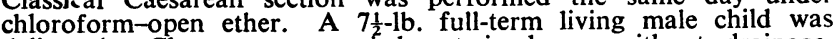
delivered. Closure was carried out in layers without drainage. Convalescence was uneventful, but there had been no bowel action by the third day. The patient then began to complain of distension and colic. The next morning she looked toxic and dehydrated, and had started vomiting. On rectal examination a la:ge smooth rounded mass was felt, firmly fixed in and filling the true pelvis. It was concluded that this was the involuting uterus, which had become retroverted and impacted in the pelvis. It was therefore decided to reopen the abdomen.

On reopening through the original incision the uterus was foun ${ }^{2}$ firmly impacted-ín the pelvis, and the pelvic colon in the region o. the pelvic-rectal junction was very congested and flattened. It was decided to perform a colostomy. A Paul's tube was inserted into the transverse colon. The uterus was then fixed with a catgut suture to the anterior abdominal wall. The obstructive symptoms passed off and the colostomy was closed four weeks later.

It may be said that at the time of the Caesarean section there had been no evidence of sacculation of the uterus, indicative of a retroversion earlier in the pregnancy.

\section{CASE II}

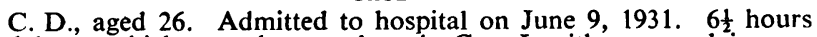
in labour, which was obstructed, as in Case I, with gross pelvic contraction. The anaesthetic and the procedure were as in Case I. An 8-lb. full-term living male child was delivered.

In this patient convalescence was from the first somewhat stormy. There were early symptoms of chest complications, but, on the other hand, in the first day or two flatus was freely passed, and no distension was observed. On the third day, however, she vomited. Vomiting continued, and she became greatly dehydrated. The usual measures were adopted without improvement. A rectal examination on the morning of June 13 again revealed a large mass impacted in on the morning of June 13 again revealed a large mass impacted in the pelvis. By this time, however, the patient was very toxic, and,
furthermore, the chest condition had developed in a manner strongly suggesting embolic phenomena. Operation being unfortunately not feasible, the patient died the next day. No necropsy was allowed, but that the mass felt was the post-partum uterus I have no doubt.

The close resemblance between this and the preceding case struck me forcibly at the time, and I felt that prophylactic ventrifixation, as employed in the first case, would have prevented the post-partum uterus falling into the contracted pelvis and obstructing the colon. I have adopted the practice of ventrifixation after Caesarean delivery in all cases since then, and no symptoms of obstruction have occurred in any of them.

\section{Discussion}

In both cases Caesarean section was performed for marked pelvic contraction. Even if a post-partum uterus were to become retroverted and fall into a normal pelvis there would be sufficient clearance to prevent its becoming impacted there. In the Caesarean operation the uterus is firmly compressed manually. Perhaps, in so doing, it is made small enough to fall into the contracted true pelvis, given that the latter is grossly contracted.

Whatever is the precise pathology, I am convinced that in bcth these cases the cause of the obstruction was the physical 
mass of the retroverted post-partum uterus impacted in the (contracted) true pelvis.

\section{OBSERVATIONS}

Inpaction of the retroverted post-partum uterus in the concrrcted true pelvis has never, so far as I have been able to determine, been quoted as a cause of intestinal obstruction after Caesarean delivery.

Having seen it occur twice, it has seemed wise and logical to perform prophylactic temporary ventrifixation in all my subsequent cases of Caesarean section. Since adopting this practice I have seen no suggestion of obstructive symptoms in any of these cases of Caesarean section, some 20 in number. It would be absurd, however, to argue from two or twenty cases.

The mechanical obstruction I have described may be operative in a proportion of cases hitherto diagnosed as post-operative "ileus." Spontaneous remission would of course be expected in most cases, as the uterus involuted.

I am much indebted to Mr. T. J. Shields, the B.M.A. librarian, for supplying the references to this paper.

\section{W. Hilton Parry, M.C., M.B., Ch.B.} REFERENCES

Haultain, W. F. T., Robertson, E. M., and Dewar, J. B. (1933-4). Trans. Edinb. obstet. Soc., 54, 121.

Montgomery, T. L. (1936). Amer. J. Obstet. Gynec., 31, 968.

O'Connor, C. T. (1934). New' Engl. J. Med., 210, 948.

\section{Pediculosis Capitis and Corneal Lesions}

The object of this memorandum is to draw attention to the frequency of severe corneal lesions associated with pediculosis capitis. It is a summary of observations made on 25 patients admitted to hospital for corneal lesions. I have been unable to trace any similar condition mentioned in the literature available to me, and I should be interested to know whether this observation has been made elsewhere.

It is, I think, a matter of common experience in wartime to find an increasing number of out-patients infected with lice. These observations were made in the out-patient department of the Infirmary during the last six months, with an average 200 patients a day. These infected patients, who came to us for eye consultation, were mostly young children, well fed, without apparent signs of general debility. The presence of lice, which were invariably Pediculus capitis, was discovered by chance on examination or later in the wards after admission. We could never find any phthiriasis palpebrarum, and the cilia were always unaffected. Apart from the conjunctivitis which was always present, the majority of cases had corneal ulcers (14 cases), 8 cases marginal keratitis, 1 case hypopyon ulcer, and 2 cases severe conjunctivitis without corneal lesion. The age incidence was as follows: 20 patients under 10 years and 5 patients over 10 years. The oldest patient was a woman aged 30 . The Wassermann reaction was negative in all but one case.

The patients usually complained of burning pain of short duration in one eye, which gradually became worse and was accompanied by photophobia. The lesion was always unilateral. A severe conjunctivitis with some muco-purulent discharge was invariably present. Bacteriological examination of the conjunctival sac showed nothing characteristic; diphtheroid bacilli, streptococci, and staphylococci were present in some cases.

The corneal ulcers had no special features; they were central or paracentral infiltrations in the substantia propria with loss of epithelium. The marginal keratitis consisted of multiple subepithelial infiltrations with a quick tendency to break down and to form confluent paramarginal crescentic ulcers. In some cases they were confined to one side of the cornea only; in other cases they tended to spread all round the corneal margin, thus forming a more or less complete ring of infiltration. Slitlamp examination showed infiltration of the anterior part of the substantia propria. Iris irritation was often present, but no K.P. or posterior synechiae were ever observed. Although this condition is very similar to the metastatic marginal ulcer of Fuchs, it showed but little tendency to respond to the routine treatment. In fact, it took a long time to heal, during which period the eye remained very congested.

The routine treatment was a sassafras cap for the head, carbolization of the ulcer, atropine $1 \%$, argyrol $10 \%$, sulphapyridine or uleron tablets, and protein shock (milk injections).

It seems probable that lice infestation causes not only conjunctival irritation but lowers the vitality of the cornea, resulting in severe corneal infiltration with little tendency to heal.

My thanks are due to the honorary surgeons of the Wolverhampton and Midland Counties Eye Infirmary-Mr. G. F. Haycraft and Mr. H. Campbell Orr-and the assistant surgeon, Mr. A. W. Green, for permission to publish this memorandum.

ARnost HiRTenstein, M.D.Prague.

Wolverhampton and Midland Counties Eye Infirmary.

\section{Reviews}

\section{THE CAMPAIGN AGAINST TUBERCULOSIS}

The Modern Attack on Tuberculosis. By Henry D. Chadwick, M.D., and Alton S. Pope, M.D. (Pp. 95. 6s.) New York: The Commonwealth Fund : London: Oxford University Press. 1942.

Drs. Chadwick and Pope detect in most works on tuberculosis a singular tendency to look on the disease as an isolated problem rather than as one of the communicable diseases in its total social and economic background. Since enough knowledge is already available to make the eradication of tuberculosis possible within a few generations if the established techniques are effectively applied, they have set out to provide " a concise digest of the experience of many workers and of present-day practices in a form serviceable to the health officer and administrator." The attempt has been wholly successful, and we strongly recommend this little book to readers even in this country, and not only to medical officers of health but to tuberculosis officers and medical superintendents of sanatoria,- who will find among its contents sound principles and common-sense conclusions that will act as salutary correctives to preconceived notions or those that are merely the fashion of the moment.

In the U.S.A. sanatorium beds have increased from 9,000 to 90,000 in 40 years. Tuberculosis in dairy cattle has been almost eliminated, and pasteurization is widespread. But, unfortunately, as in this country, little progress has been made in finding cases of early tuberculosis. In naming the essentials of a programme for the eradication of the disease, the authors therefore put first "a medical profession interested in the problem and familiar with modern methods of diagnosis and treatment." And they stress the importance of a knowledge of certain basic data before embarking on any sound antituberculosis campaign. The various diagnostic procedures are critically examined, and the conclusion at the end of this chapter deserves emphasis: "Prompt diagnosis is important to the patient, to the family, to the community. Every suspected case of tuberculosis should be cleared of suspicion as quickly as possible by being proven innocent or guilty of harbouring the disease. Time-consuming observation by physicians without resort to all the diagnostic aids at our command is to be condemned and rated as a disservice to the patient that borders on malpractice."

The sections on the sanatorium as a means of control and treatment, and on case finding in the community, are no less interesting and no less well balanced. The authors hold that tuberculosis presents one of the hardest administrative problems that confronts the health officer. They emphasize that when an authority fails to provide enough beds it is committed to a plan that is wasteful, ineffective, and in the long run costly. "Half-way measures will not exterminate tuberculosis. A sanatorium bed should be available for every active case of tuberculosis regardless of the stage of the disease. Treatment that will benefit the patient and also serve to shield others by protecting them from infection is the real objective of case finding." And, to quote again from later in the book: "From the standpoint of case finding the shift in the age distribution of tuberculosis mortality means that greater emphasis must be placed on the examination of adult contacts and on mass examinations of groups subject to the highest risks, such as workers in hazardous industries and adults on relief rolls." Patients in ante-natal clinics should be among the first to be considered for mass examination ; and a selection of other groups should be based upon actual incidence of significant tuberculosis, as demonstrated by preliminary sampling. The yield of mass examinations will vary directly with the incidence of tuberculosis in the area concerned, and in many parts of the U.S.A. this has already fallen to a point which makes the cost of mass radiography, relative to the number of cases found, prohibitive. From the last chapter, "A Community Campaign of Eradication," we choose one final quotation:

"Too often health officers have taken the "what's-the-use" attitude in regard to tuberculosis. They have been content to make the record a mere bookkeeping chore and let the case rest there. The health officer should look upon tuberculosis as a communicable 